\title{
Intraoperative Statistical Parametric Brain Mapping using RGB Imaging
}

\author{
Charly Caredda ${ }^{a, *}$, Laurent Mahieu-Williame $^{a}$, Raphaël Sablong ${ }^{a}$, Michaël Sdika ${ }^{a}$, \\ Jacques Guyotat ${ }^{b}$ and Bruno Montcel ${ }^{a, *}$ \\ ${ }^{a}$ Université de Lyon, INSA-Lyon, Université Claude Bernard Lyon 1, UJM-Saint Etienne, CNRS, Inserm, \\ CREATIS UMR 5220, U1206, F69100 Lyon, France \\ ${ }^{b}$ Service de Neurochirurgie D, Hospices Civils de Lyon, F69500 Bron, France \\ *charly.caredda@creatis.insa-lyon.fr,bruno.montcel@creatis.insa-lyon.fr
}

\begin{abstract}
We present the methodology for the intraoperative pixel-wise identification of activated cortical areas using RGB imaging. The results indicate that RGB imaging could be a useful complement to the electrical brain stimulation. () 2021 The Author(s)
\end{abstract}

\section{Introduction}

Non invasive functional brain mapping is an imaging technique used to localize the functional areas of the patient brain. This technique is used during brain tumor resection surgery to indicate to the neurosurgeon the cortical tissues which should not be removed without cognitive impairment. Functional magnetic resonance imaging (fMRI) [1] is widely used to localize patient functional areas. However, after patient craniotomy, a brain shift invalidates the relevance of neuronavigation to intraoperatively localize the functional areas of the patient brain [2]. In order to prevent any localization error, intraoperative MRI has been suggested but it complicates the surgery gesture which makes it rarely used. For these reasons, Electrical brain stimulation (EBS) [3] is preferred during neurosurgery, but this technique is mainly limited by its low spatial resolution and has the potential risk to trigger epileptic seizures. Optical imaging provides an ideal solution for intraoperative functional brain mapping because the analysis of the light absorption allows to monitor the brain activity with quantification of the concentration changes in oxy- $\left(\Delta C_{\mathrm{HbO}_{2}}\right)$ and deoxygenated hemoglobin $\left(\Delta C_{H b}\right)$ in brain cortex [4].

During neurosurgery, the craniotomy gives a direct access to the brain cortex. Intrinsic optical imaging [5] can be used intraoperatively to localize the patient hemodynamic activity in the cerebral cortex. The intrinsic signal refers to the cortical reflectance changes due to hemodynamic response [6].

Semi-quantitative [7] or quantitative [8] techniques can be used to identify the functional cerebral zones of the patients during neurosurgery. These techniques provide to the neurosurgeon a lot of information that cannot be easily used to guide the surgical gesture. For instance, these techniques do not provide a binary identification of activated cerebral areas as provided by Statistical Parametric Mapping techniques (SPM [9]) used in fMRI and fNIRS (functional Near InfraRed Spectroscopy).

In these works, we propose the methodology to provide a binary identification of activated cerebral areas during neurosurgery inspired by fMRI SPM techniques. The results obtained with RGB imaging show a good correlation with the electrical brain stimulation identifications.

\section{Material and methods}

\subsection{Experimental Setup}

The wide field optical device employed in this study is presented in Fig. 1 and described in Ref [4]. This device is composed of a RGB camera (BASLER acA2000-165uc) in conjunction with an Edmund Optics camera lens $(f=50 \mathrm{~mm} f / 2-f / 22)$, a continuous wave white light source (OSRAM Classic 116W 230V light bulb) and a laptop (processor: Intel Core i5-7200U, $2.50 \mathrm{GHz} \times 4$, ram: $15.3 \mathrm{GiB}$ ). The camera provided 8 bits RGB images every $33 m s$. 


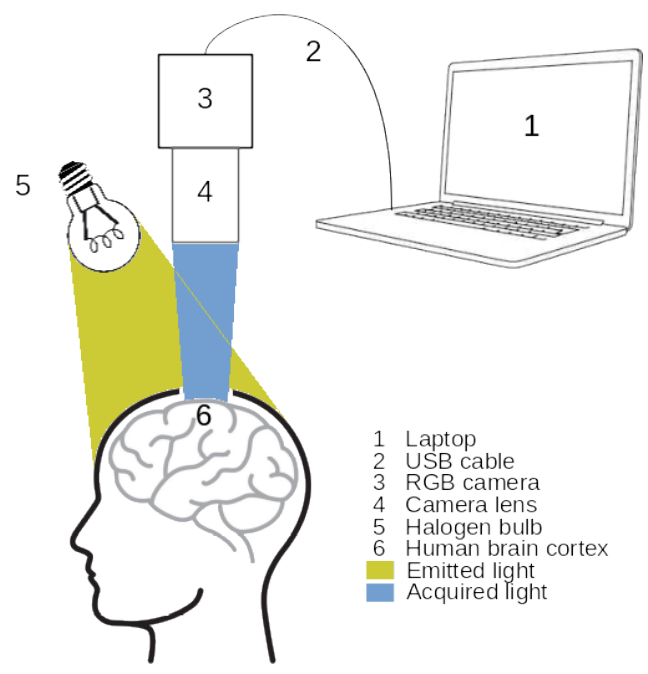

Fig. 1. Schematic of the imaging system.

\subsection{Patient Inclusion and Experimental Paradigm}

The study was conducted at the neurologic center of the Pierre Wertheimer hospital in Bron, France. A patient presenting a tumor close to the motor cortex area was included in the study (awake surgery). A 2 minute video was acquired after patient craniotomy and before brain tumor resection operation. During the acquisition, the patient motor cortex associated to hand motricity was stimulated by two 20 s periods of repetitive right fist clenching separated by rest periods. The fist clenching was performed by the patient itself. After the acquisition, the neurosurgeon operated EBS to localize the patient's brain motor area.

\subsection{Functional Model}

Once the video was acquired, the quantitative model described in Ref [4] was applied to obtain $\Delta C_{H b}$ and $\Delta C_{H_{b O}}$ time courses for each camera pixel. A general linear model was implemented to test the correlation between measured and theoretical patient hemodynamic responses. An image of $t$ statistics is thus obtained. This image is then thresholded using Gaussian random field theory [9]. This aims to identified pixels associated with an activated functional area by calculating a $t$ statistics threshold that reject the null hypothesis at $5 \%$ significance level (null hypothesis: there is no cerebral activity, i.e. measured and theoretical hemodynamic reponses do not correspond).

\section{Results and discussion}

In Fig. 2, the first image of the video sequence (image (a)) and the activation maps obtained using SPM analysis with $\Delta C_{\mathrm{HbO}_{2}}, \Delta C_{\mathrm{Hb}}$ and $\Delta C_{\mathrm{HbT}}$ maps are represented in images (b), (c) and (d) respectively. The cortical areas identified as activated in images (a), (b) and (c) correspond to the motor area identified by electrical stimulation (letter M).

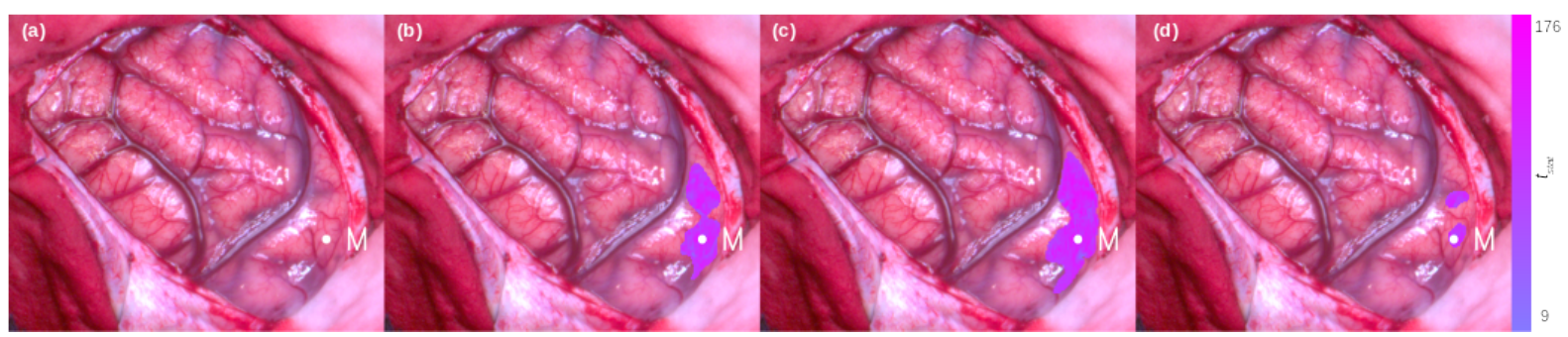

Fig. 2. (a) First image of the video sequence. The white spot and the letter $\mathbf{M}$ indicate the motor area identified by electrical brain stimulation. (b) $\Delta C_{\mathrm{HbO}_{2}}$ activation map. (c) $\Delta C_{H b}$ activation map. (d) $\Delta C_{H b T}$ activation map. The colorbar represents the $t$ statistics range of variation.

The $\Delta C_{H b}$ activation map is more extended than the two other maps. This differences may be explained by the definition of our theoretical hemodynamic response. We used the BOLD signal used in fMRI studies that 
is predominately due to the paramagnetic properties of deoxy-hemoglobin. We also observe that the $\Delta C_{H b T}$ activation map allows a much more localized identification than the two other maps that is consistent with Culver et al. works [10].

This functional model could be improved. For this purpose, the patient's oxy- and deoxygenated hemodynamic response function to an impulse stimulus could be experimentally measured. Indeed, the hemodynamic response functions are patient dependant since these functions differ depending on the type of cortical tissue (grey matter,arterioles) [11]. It has also been shown that the neurovascular system evolves with age, which implies a change in hemodynamic response [12]. Moreover, Montgomery et al. [13] showed that the, progression of gliomas over time implies a change in the hemodynamic response.

\section{Conclusion}

The current work demonstrates that an RGB camera combined with a quantitative modeling of brain hemodynamics biomarkers could evaluate in a robust way the functional areas during neurosurgery. Moreover, this work indicates that RGB imaging combined with a SPM analysis could be a useful tool to complement the EBS during neurosurgery. This strengthens the relevance of using a classical RGB camera for functional intraoperative brain imaging.

\section{Acknowledgments}

These works were funded by LABEX PRIMES (ANR-11-LABX-0063) of Université de Lyon, within the program "Investissements d'Avenir" (ANR-11-IDEX-0007), operated by the French National Research Agency (ANR); Cancéropôle Lyon Auvergne Rhône Alpes (CLARA) within the program “OncoStarter"; Infrastructures d'Avenir en Biologie Santé (ANR-11-INBS-000), within the program "Investissements d'Avenir" operated by the French National Research Agency (ANR) and France Life Imaging (ANR-11-INBS-0006). We want to acknowledge the PILoT facility for the support provided for the image acquisition.

\section{References}

1. S. Ogawa et al. Brain magnetic resonance imaging with contrast dependent on blood oxygenation. Proceedings of the National Academy of Sciences, 87(24):9868-9872, December 1990.

2. Ian J. Gerard et al. Brain shift in neuronavigation of brain tumors: A review. Medical Image Analysis, 35:403-420, January 2017.

3. Wilder Penfield et al. Somatic motor and sensory representation in the cerebral cortex of man as studied by electrical stimulation. Brain, 60(4):389-443, 1937.

4. Charly Caredda et al. Intraoperative quantitative functional brain mapping using an RGB camera. Neurophotonics, 6(4):1 - 14, 2019.

5. A. Grinvald, E. Lieke, R. D. Frostig, C. D. Gilbert, and T. N. Wiesel. Functional architecture of cortex revealed by optical imaging of intrinsic signals. Nature, 324:361-364, November 1986.

6. Katherine A. Morone, Joseph S. Neimat, Anna W. Roe, and Robert M. Friedman. Review of functional and clinical relevance of intrinsic signal optical imaging in human brain mapping. Neurophotonics, 4(3):031220, June 2017.

7. Martin Oelschlägel et al. Mapping of language and motor function during awake neurosurgery with intraoperative optical imaging. Neurosurgical Focus FOC, 48(2):E3, 01 Feb. 2020.

8. Matthew B. Bouchard et al. Ultra-fast multispectral optical imaging of cortical oxygenation, blood flow, and intracellular calcium dynamics. Optics Express, 17(18):15670, August 2009.

9. K. J. Friston, editor. Statistical parametric mapping: the analysis of funtional brain images. Elsevier/Academic Press, Amsterdam ; Boston, 1st ed edition, 2007.

10. Joseph P. Culver et al. Evidence that cerebral blood volume can provide brain activation maps with better spatial resolution than deoxygenated hemoglobin. NeuroImage, 27(4):947-959, October 2005.

11. Bruyns-Haylett et al. Temporal coupling between stimulus-evoked neural activity and hemodynamic responses from individual cortical columns. Physics in Medicine and Biology, 55(8):2203-2219, April 2010.

12. Michele Veldsman, Toby Cumming, and Amy Brodtmann. Beyond BOLD: Optimizing functional imaging in stroke populations: Optimizing BOLD Imaging in Stroke. Human Brain Mapping, 36(4):1620-1636, April 2015.

13. Mary Katherine Montgomery et al. Glioma-Induced Alterations in Neuronal Activity and Neurovascular Coupling during Disease Progression. Cell Reports, 31(2):107500, April 2020. 